\title{
Evaluación, avalúo y ciclo de vida de la tecnología (Parte II)
}

\author{
L.R. Vega-González \\ Centro de Ciencias Aplicadas y Desarrollo Tecnológico, UNAM \\ Coordinación de Vinculación, Laboratorio de Cibernética y Sistemas \\ E-mail:lrvg@servidor.unam.mx
}

(recibido: junio de 2001; aceptado: diciembre de 2002)

\begin{abstract}
Resumen
Uno de los aspectos determinantes en una evaluación tecnológica es la determinación del precio o avalúo. Este es un proceso complejo y muchas veces incierto, ya que existe diversa literatura y referencias al respecto, pero su abundancia resulta confusa cuando no se tiene un punto de referencia. La propuesta metodológica de la segunda parte de este artículo es, en primer término, identificar la posición que guarda la tecnología dentro de su ciclo de vida para posteriormente utilizar las técnicas de valoración más adecuadas según el caso o zona de incertidumbre. Este trabajo recupera la experiencia de varios años valorando tecnologías para negociar su transferencia en la Universidad Nacional Autónoma de México (UNAM), así como en el caso de proyectos de desarrollo de empresas privadas.
\end{abstract}

Descriptores: valuación de tecnología, precio de la tecnología, ciclo de vida y transferencia de tecnología.

\begin{abstract}
One of the main aspects in a technological evaluation is the pricing determination or valuation. This is a complex an uncertain process. There are different kinds of literature and references talking about this topic but its abundance results in confusion when there is no an initial point of departure. The methodological proposal presented in this second article part, calls in first place for identifying the technology position against its life cycle for the later use of the ap. propriated valuation techniques depending on the case or uncertainty zone. This work recovers the experience of several years pricing technologies for technology transference at the National Autonomous University of Mexico (UNAM) and other private firms development projects.
\end{abstract}

Keywords: technology valuation, technology pricing, life cycle, and technology transfer.

\section{Importancia del avalúo tecnológico}

La determinación del precio de una tecnología o avalúo tecnológico es un asunto de interés para todos aquellos que desarrollan, producen, venden y compran tecnología o productos tecnológicos. Como usuarios, prácticamente todos nos hemos visto en la necesidad de estimar el precio más adecuado para una nueva computadora, un auto o algún artefacto doméstico. En 
las empresas u organizaciones, el personal técnico de supervisión, mantenimiento y los niveles directivos, deben tener alguna sensibilidad del costo que representará la compra de un nuevo equipo, de unidades de proceso, o de herramientas especiales, ya sea para alguna unidad de producción nueva, o para modernizar una sección de la planta.

En general, podemos decir que en las organizaciones la valuación tecnológica es una actividad directiva crítica que sirve como soporte para la toma de decisiones y que tiene grandes implicaciones operacionales y estratégicas (Shippley y Jobber, 2001).

Un avalúo tecnológico se puede realizar como parte integrante de un proceso de evaluación tecnológica más amplio o como un proceso independiente.

En la primera parte de este artículo, publicado en esta revista en el número anterior de julio-septiembre 2003, se hizo énfasis en que la valoración de la tecnología es parte del método de evaluación tecnológica determinada por cuatro etapas: (a) aspectos críticos, (b) grado de integración del paquete tecnológico, (c) índice de desempeño globaly (d) precio de la tecnología.

Existen muchos trabajos de valuación tecnológica que cubren una extensa gama de métodos. Entre los más comunes se tienen la generación de tablas de ponderación de factores (Rodríguez y Solleiro, 1991) y las clásicas valuaciones de tipo financiero, como el análisis costo beneficio o las basadas en costos (Martin, 2000), (Willis et al., 1998). Para casos más complejos como el manejo de ambientes de incertidumbre, en los que con el tiempo existe volatilidad de precios, se utilizan teorías numéricas especiales como la de opciones de valor (Newton y Pearson, 1994), los sistemas integrales de evaluación (Premachandraetal., 2000), la valuación de contingencias (Whittington, 1998), (Blumeschein y Johannesson, 1999) y recientemente la valuación ecológica (Drucker et al., 2001), (Stirling, 1997), por citar unos cuantos ejemplos.

La diversidad es muy grande y tiende a confundir al analista. Evidentemente afloran muchas preguntas como: ¿Cuándo debo usar un tipo de metodología de valoración?, ¿Es el método más apropiado?, ¿Cuál es el fundamento de mi propuesta de avalúo?, entre muchas otras.

Para reseponder estas preguntas, más adelante se propondrá un método de avalúo tecnológico en el que el requisito inicial es identificar cuál es la posición de la tecnología que deseamos valorar, respecto a su ciclo de vida.

\section{Análisis del ciclo de vida de una tecnología}

El ciclo de vida es un concepto que ha sido estudiado intensamente desde los años sesenta por los teóricos de la mercadotecnia como Levitt (1965), Pride y Ferrel (1986), Schewe y Smith (1988) y Kotler (1985), entre muchos otros.

El concepto se refiere básicamente a productos de alto consumo, dado que la tecnología puede ser en sí misma un producto. También se liga al hecho de que los productos incorporen tecnología en su fabricación, lo que puede extender el concepto y hablar del ciclo de vida de los productos tecnológicos o del ciclo de vida de la tecnología. La función $Y_{t}$ de la figura 1 , muestra las fases del ciclo de vida de una 
tecnología. A la función $Y_{t}$ se le conoce como curva de difusión, curva logística o curva "S". Generalmente, antes de que un producto tecnológico ingrese en la fase de declinación, el fabricante, como parte de sus estrategias de mercadeo y control de mercados, modifica el producto, lo cual puede originar a innovaciones graduales o radicales.

A las curvas de difusión también se les conoce como curvas logísticas. La figura 2 muestra un buen ejemplo de sucesión de tecnologías.

Las unidades de medición de $Y_{t}$ pueden ser el número de unidades que se han difundido o que se encuentran en el mercado; o también el margen de utilidad que han generado las ventas de la tecnología en cuestión. Es natural que para efectos de avalúo nos interesen las unidades monetarias del margen de utilidad.

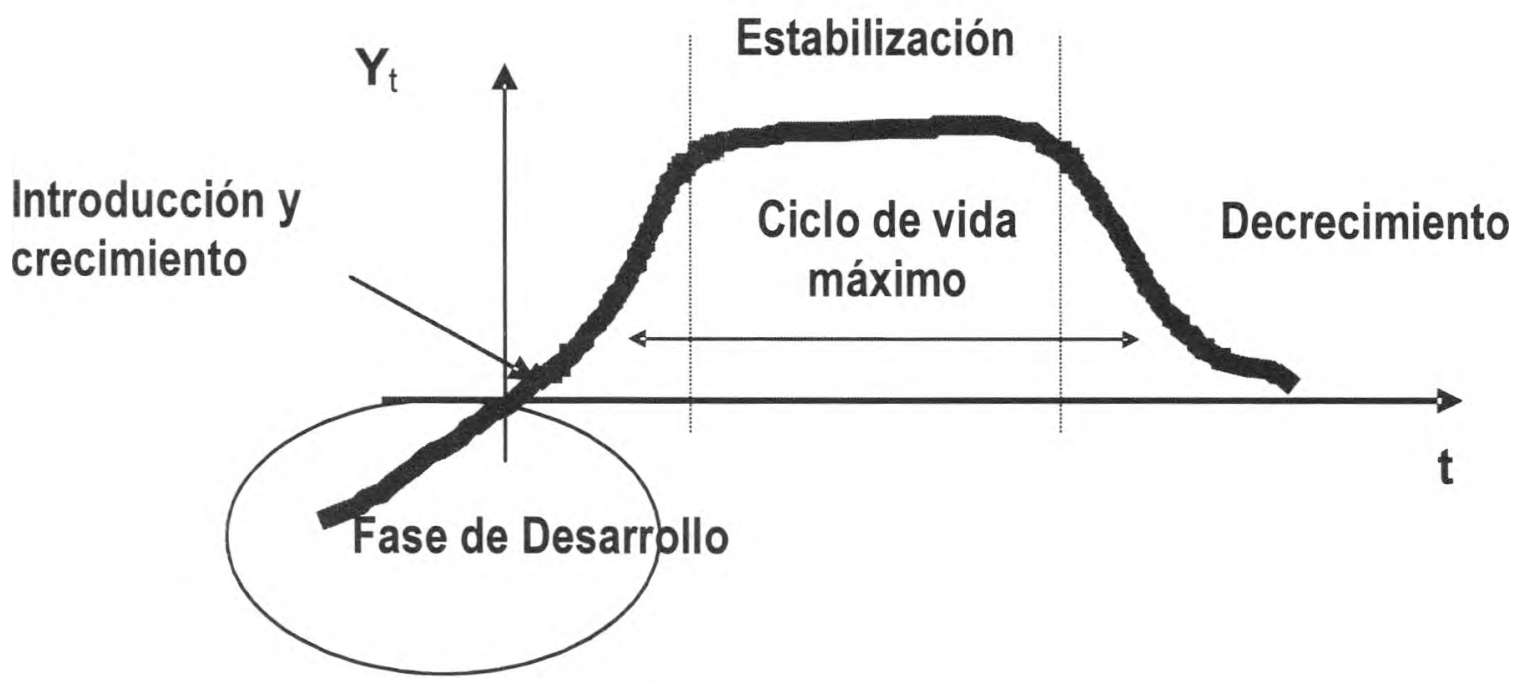

Figura 1. Ciclo de vida de la tecnología

(Ptride y Ferrel,1986); (Schewe y Smith, 1988)

\section{$Y_{\mathrm{t}}$ Difusión}

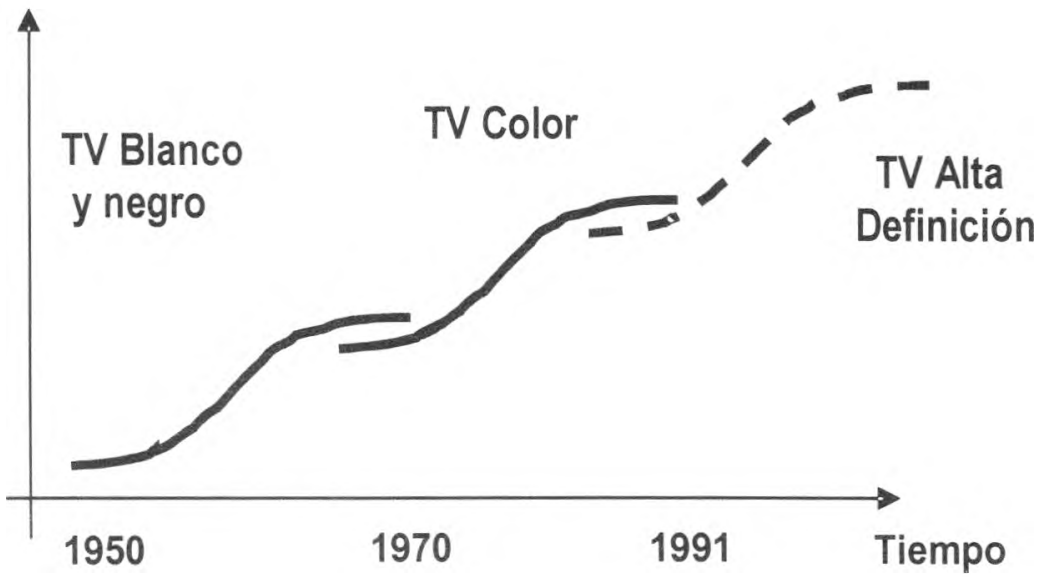

Figura 2. Sucesión de curvas de difusión de productos tecnológicos

Fuente: UNIDO (1996) 
Existen diferentes modelos matemáticos de las curvas de difusión. Los modelos más comunes utilizan funciones logaritmicas o exponenciales (Everett,1995). En algunos casos, es posible utilizar funciones senoidales.

La figura 3 muestra un modelo "suavizado" del ciclo de vida del tipo senoidal. La función tiene "offset", es decir, está fuera de centro porque el valle representa las inversiones, costos o utilidades negativas que se requieren durante la fase de desarrollo de la tecnología. La cresta es mayor porque se supone que el potencial de utilidades será muy superior a lo invertido. Se dice que en la fase de desarrollo las tecnologías son precompetitivas, ya que aún no generan ingresos, y por lo tanto, tampoco utilidades.

Analizando el ciclo de vida desde el punto de vista de la variable horizontal tiempo encontramos cuatro etapas en la vida de un producto tecnológico: desarrollo, introducción y crecimiento, estabilización y decrecimiento.

Cuando se analiza la función respecto a la ordenada, se encuentra una situación diferente, ya que se desconoce el valor que tomarán las utilidades en cada una de las etapas de desarrollo de la tecnología, por lo que nos encontramos con tres zonas bien delimitadas de incertidumbre.

\section{Definición de zonas de incertidumbre en el ciclo de vida}

La figura 4 muestra la primera zona denominada de máxima incertidumbre en el ciclo de vida. La incertidumbre máxima radica en que se trata del estado precompetitivo de la tecnología, por lo que se desconocen los costos de desarrollo total, los costos de escalamiento y su factibilidad técnica total.

No existe una técnica con exactitud y certeza de estimación de costos para proyectos de nueva tecnología (Colmer et al., 1999). También se desconoce la situación del mercado potencial, la aceptación real del mercado y la percepción pública. No hay datos financieros estadísticos como los estados de resultados, las tasas de rentabilidad ni flujos de efectivo.

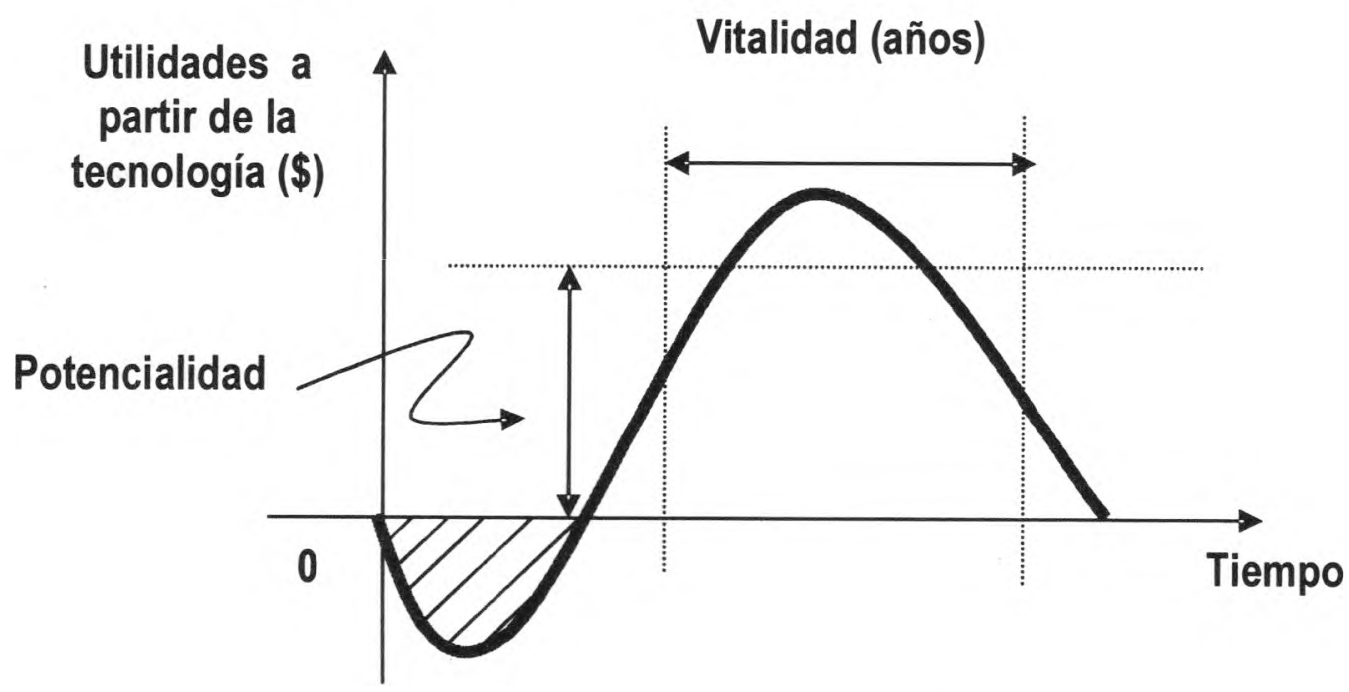

Figura 3. Modelo suavizado del ciclo de vida 


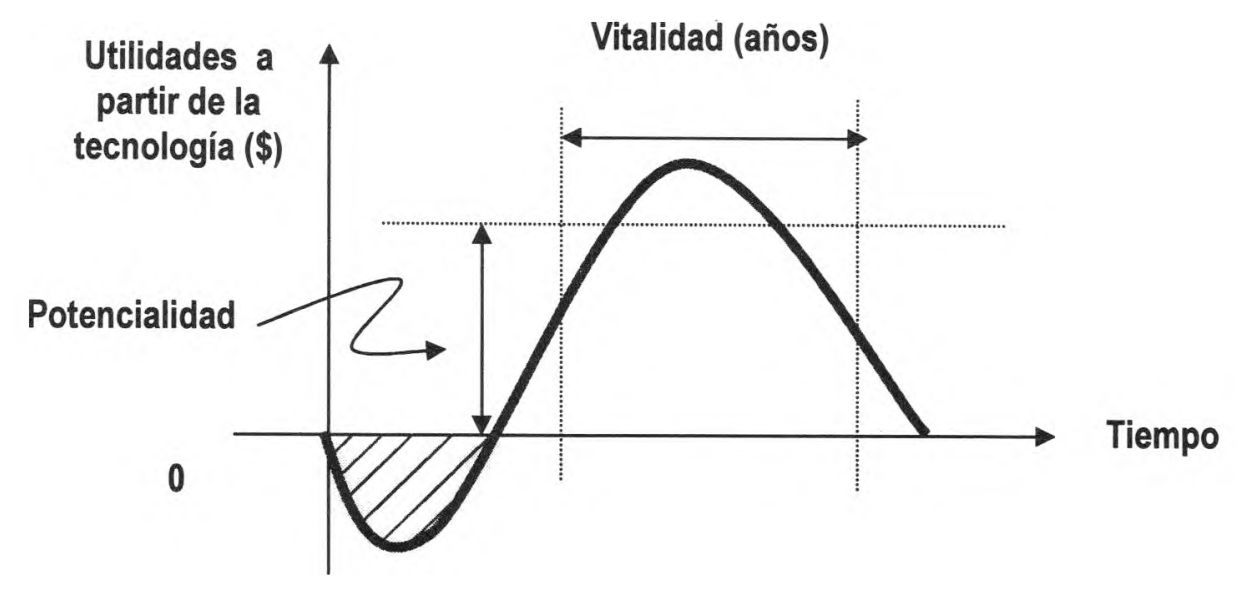

Figura 4. Zona 1. Incertidumbre máxima

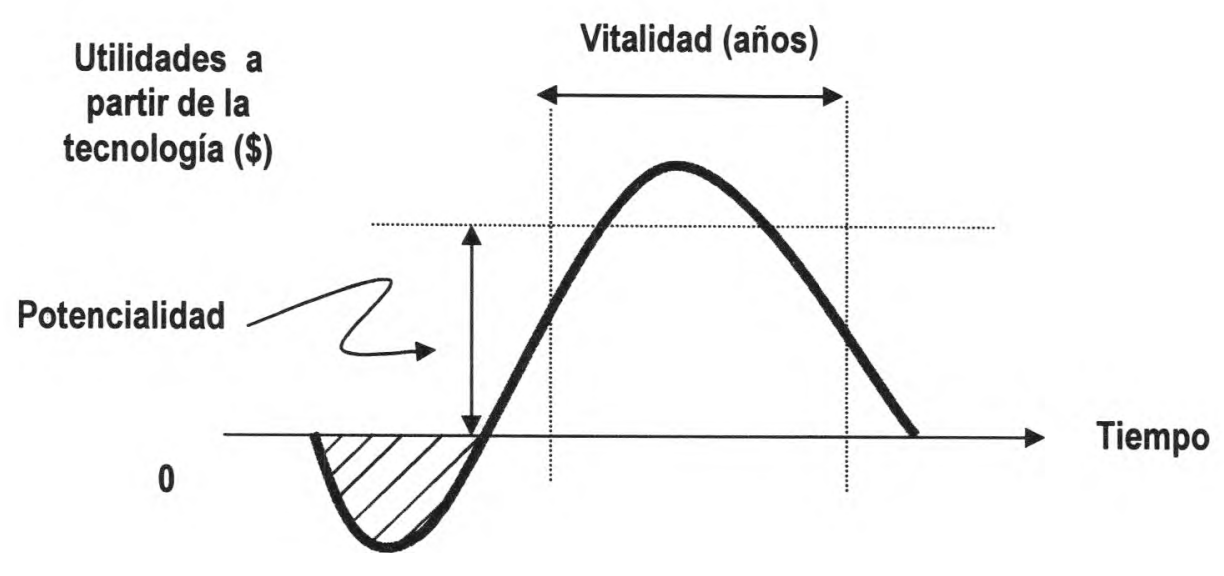

Figura 5. Zona 2. Incertidumbre moderada

Abreviando, en esta zona sólo existen grandes expectativas de lo que sucederá con el producto tecnológico en el mercado, por lo que las técnicas de valuación se basan en estimaciones y especulación.

La figura 5 muestra la zona 2 de incertidumbre moderada caracterizada, ya que la tecnología entra en un estado de competencia incipiente. En esta zona ya se conocen los costos de desarrollo hasta el nivel de prototipos; sin embargo, los costos de escalamiento no se tienen en su totalidad debido a que se están depurando los procesos productivos.

La factibilidad técnica total dependerá de la implantación de un buen sistema de asimilación y adaptación de la tecnología, que evidentemente agrega costos al proceso de escalamiento industrial.

La penetración en el mercado potencial se encuentra en desarrollo, lo cual es muy costoso porque requiere de promoción, per- 
sonal de ventas y la generación de una imagen pública.

La estadística de datos financieros sigue siendo insuficiente, pero los datos se pueden proyectar considerando la poca historia de las ventas y las tendencias esperadas de crecimiento a través de pronósticos de ventas.

En resumen, la tendencia de comportamiento de la elevación y la baja de la función es un poco menos especulativa. En la primera ya se cuenta con datos de costos y se inicia el perfil de las tendencias de comportamiento del mercado y financieras.

La baja de la incertidumbre la determina el desconocimiento del valor de la pendiente de bajada o de la disminución del mercado. Esto no puede pronosticarse con precisión, ya que depende del estado de la nueva competencia, de la percepción e intereses del mercado, y por supuesto, del mismo cambio tecnológico.

La figura 6 muestra la zona 3 de incertidumbre mínima, caracterizada porque la tecnología entra en un estado de franca competencia. Este es un período de estabilización o explotación de la vitalidad en el que ya se conocen plenamente los costos de producción y se busca la optimización de la productividad.

El sistema de asimilación y adaptación de la tecnología se encuentra probado. Se cuenta con un segmento del mercado establecido y una imagen pública. Generalmente, en esta zona el comportamiento de la tendencia de los mercados puede ser pronosticable con mayor éxito que en las otras dos zonas. Desde el punto de vista financiero, existen datos históricos de ventas, con los cuales se pueden realizar cálculos y proyecciones de corto y mediano plazo.

Concretando, en esta zona el comportamiento de los productos tecnológicos es predecible, pero existe el riesgo de que termine el ciclo de vida

\section{Metodología de avalúo}

En general, un avalúo tecnológico se realiza como antecedente a un proceso de negociación para la compra o venta de tecnología. Razón por la cual el fundamento de la metodología de avalúo aquí

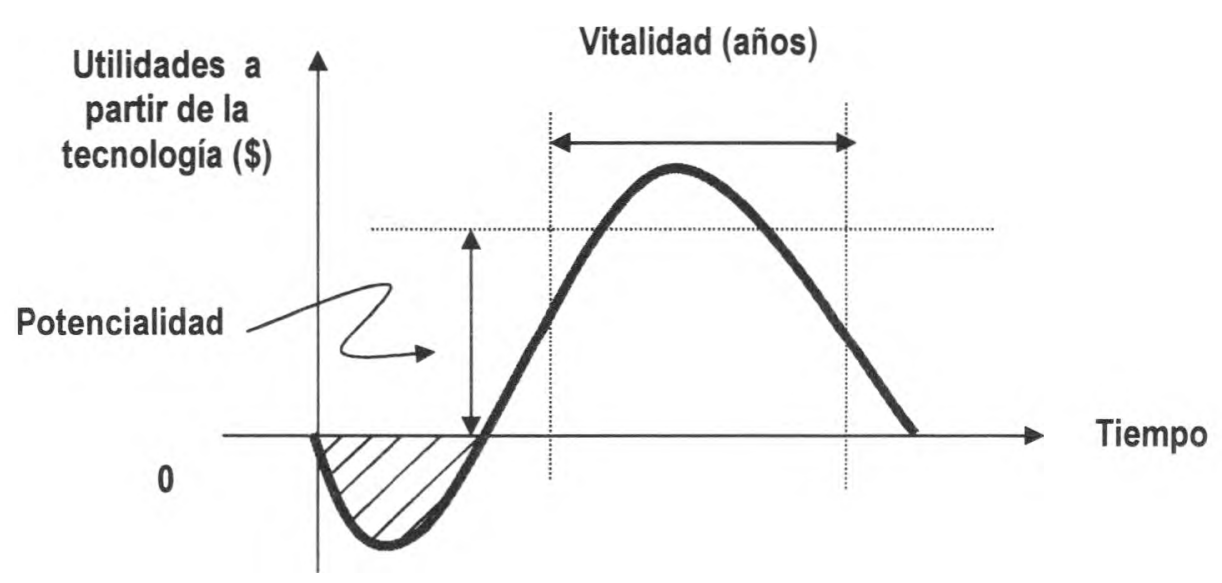

Figura 6. Zona 3. Incertidumbre mínima 
presentada, es que un avalúo de tecnología debe funcionar como una balanza entre las utilidades o beneficios esperados y los costos y/o riesgos involucrados en la transacción.

La adquisición de productos tecnológicos lleva consigo un gran riesgo natural. Puede ser que el paquete tecnológico no cubra las necesidades de la parte que adquiere la tecnología, y en consecuencia, se deban hacer erogaciones superiores para complementar el paquete, lo que además propiciará la pérdida de tiempo y materiales de producción.

Otra posibilidad se muestra cuando hablamos de productos tecnológicos con una gran cantidad de tecnología incorporada. En este caso se desconoce cuál será la aceptación del mercado por lo que se puede incurrir en pérdidas sobre las inversiones en equipos, maquinaria e inventarios.

Para disminuir el efecto del riesgo, desde hace más de tres siglos se adoptó el mecanismo de pago de regalías que lleva implícito el concepto de riesgo compartido.
A la fecha, la forma de pago universal de las tecnologías es a través de regalías. Este pago se define a través de un cálculo periódico, ya sea anual o semestral sobre la base de un indicador de desempeño del proceso de comercialización (Bidault, 1989), (WRF, 1992).

Las regalías generalmente se estiman como un porcentaje de las ventas o de las utilidades obtenidas por la difusión y uso de la tecnología. De esta forma, el riesgo se comparte entre los que adquieren y los que venden la tecnología. El concepto central es que la parte que desarrolla y vende la tecnología sólo recibirá pago si hay ventas.

Existen muchos otros mecanismos de pago como los que se realizan por adelantado y en efectivo, un solo pago, un pago y regalías, pagos en efectivo diferidos, pagos anuales durante un cierto tiempo y pago de regalías durante otro período, por citar unos cuantos ejemplos. El tipo de pago depende de la negociación que se realice. La figura 7 muestra el método de valuación propuesto referido al pago de regalías básico.

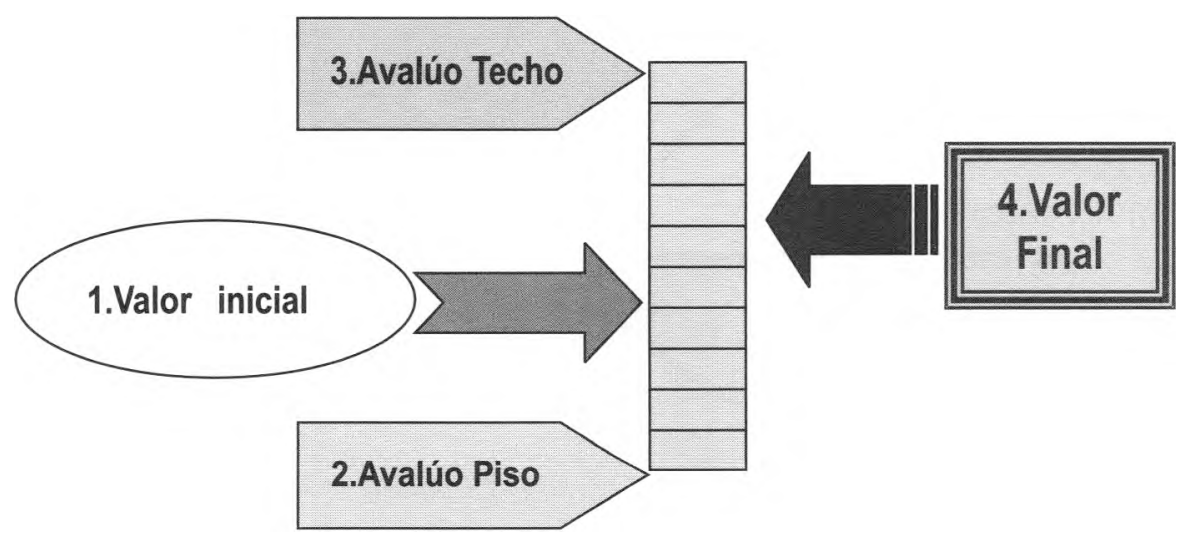

Figura 7. Metodología de avalúo 
El método de valoración se desarrolla en cuatro pasos. Primero, se determina un valor incial o semilla. Posteriormente, se fijan los limites de negociación o del valor de la tecnología. Por un lado está el valor piso, definido como el mínimo posible que podemos aceptar o pagar, por otro, está el límite superior o valor techo, definido como el precio máximo que estamos dispuestos a pagar o que esperaríamos por la tecnología. El cuarto paso es la definición del valor final a través de algunos procedimientos de ajuste. Este es el ajuste fino del valor del producto tecnológico.

\section{Paso 1. Definición del valor inicial}

La figura 8 muestra las tres alternativas más comunes utilizadas por Obrad (1992) para la definición del valor inicial o semilla.

\section{A) Criterio del 25\% de las utilidades}

Esta alternativa se refiere a la experiencia práctica internacional en porcentajes de regalías en licenciamientos. Se sabe por consulta a expertos de Estados Unidos, Canadá y las experiencias de contratos de transferencia de tecnología en México, que:

- Para bienes patentados listos para el mercado se cobra entre el 10 y el $20 \%$ de las ventas.

Para bienes patentados con prototipo se requiere entre 5 y $15 \%$ de las ventas.

En negociaciones de tecnologías incipientes con prototipos no terminados el promedio requerido es del $5 \%$ de las ventas.

$\mathrm{Si}$ las tecnologías tienen gran potencial de mercado en etapas tempranas de investigación se cobra entre 1 y $5 \%$ de las ventas

El desarrollo de Software requiere entre 10 y $15 \%$ de las ventas.

Comúnmente se habla de la regla mágica del 5\%; en general 5\% de las ventas representa entre un 20 a $25 \%$ de las utilidades totales por la venta de productos tecnológicos.

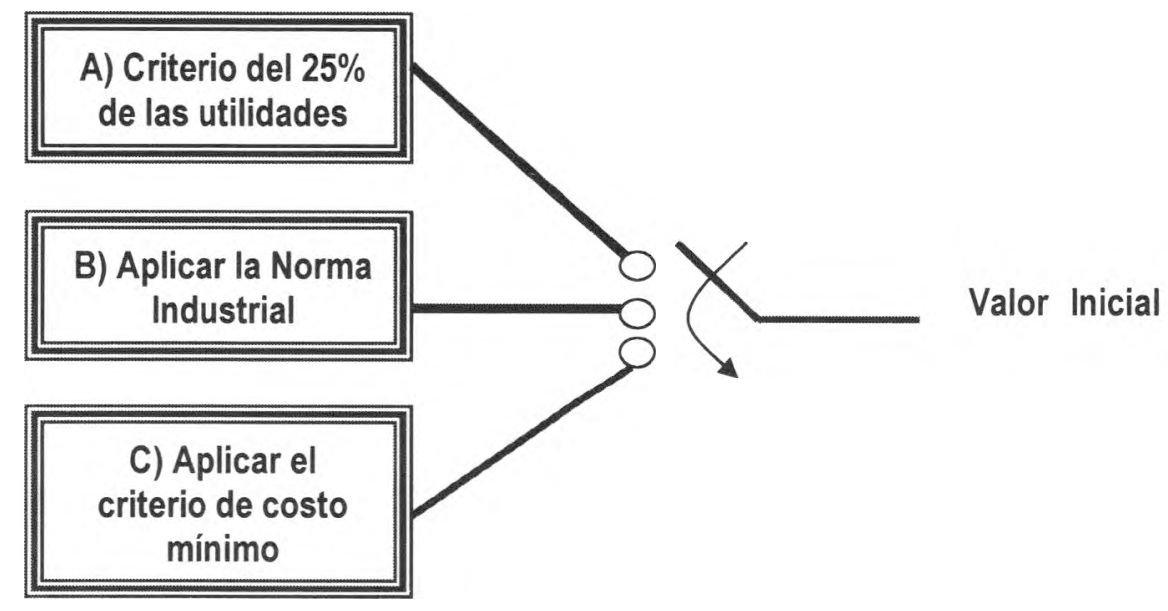

Figura 8. Alternativas para la definición del valor inicial 


\section{B) Aplicación de la norma industrial}

Entendemos como norma industrial a los criterios determinados y publicados por entidades gubernamentales y asociaciones o cámaras industriales. Se basa en datos estadísticos, típicamente en tablas de frecuencia. Existen publicaciones periódicas que son buenos auxiliares para quien practica el avalúo comolas revistas Licensing Law \& Business Report, Licensing Economics Review y Les Nouvelles, las cuales publican bimestralmente los promedios de porcentajes de regalías, cobrados según diferentes tipos de industria (Sullivan, 1994).

La tabla 1 muestra este tipo de reportes, desde el punto de vista del estadístico moda. El criterio más común en las negociaciones es del 2 al 5\%, lo que coincide con la regla mágica de la alternativa A. Incluso en el caso de la industria de alimentos de los Estados Unidos de
Norteamérica, el 100\% de las transacciones serealizan dentro de esterango deregalías.

C) Aplicación del criterio del costo minimo

Este es un criterio muy simple, consiste en que el precio de la tecnología debe ser al menos la suma total de los costos involucrados en su desarrollo. El criterio de pago podría ser en este caso, un pago único que incluyera el total de costos más algún tipo de utilidad.

\section{Paso 2. Avalúo piso}

La figura 9 muestra el proceso de cálculo para definir el mínimo valor aceptable para la tecnología. La diferencia con respecto a la alternativa $\mathrm{C}$ del precio inicial es que no considera el total de costos de desarrollo, sino que parte del supuesto de que los costos de desarrollo no pueden ser cobrados al cliente por razones muy diversas.

Tabla 1. Estándares de pago de regalías por industria en los EUA.

(McGavock et al., 1992) y (Yamasaki, 1996)

\begin{tabular}{|c|c|c|c|c|}
\hline Porcentaje de regalías $(\%) \rightarrow$ & 0 a $2 \%$ & 2 a $5 \%$ & 5 a $10 \%$ & 10 a $15 \%$ \\
\hline \multicolumn{5}{|l|}{ Tipo de Industria $\downarrow$} \\
\hline Automotriz & $52 \%$ & $45 \%$ & $3 \%$ & \\
\hline Química & $17 \%$ & $58 \%$ & $24 \%$ & $1 \%$ \\
\hline Computadoras & $62 \%$ & $31 \%$ & $6 \%$ & \\
\hline Electrónica & & $50 \%$ & $25 \%$ & $25 \%$ \\
\hline Manufacturas & $45 \%$ & $27 \%$ & $12 \%$ & $14 \%$ \\
\hline Alimentos & & $100 \%$ & & \\
\hline Aeroespacio & $52 \%$ & $50 \%$ & & \\
\hline Energía & & $67 \%$ & & \\
\hline Gobierno y Universidades & $25 \%$ & $25 \%$ & $50 \%$ & \\
\hline Equipo médico & $3 \%$ & $52 \%$ & $45 \%$ & \\
\hline Farmacéuticos & $24 \%$ & $32 \%$ & $29 \%$ & $13 \%$ \\
\hline Reactivos de diagnóstico & & $50 \%$ & $50 \%$ & \\
\hline
\end{tabular}




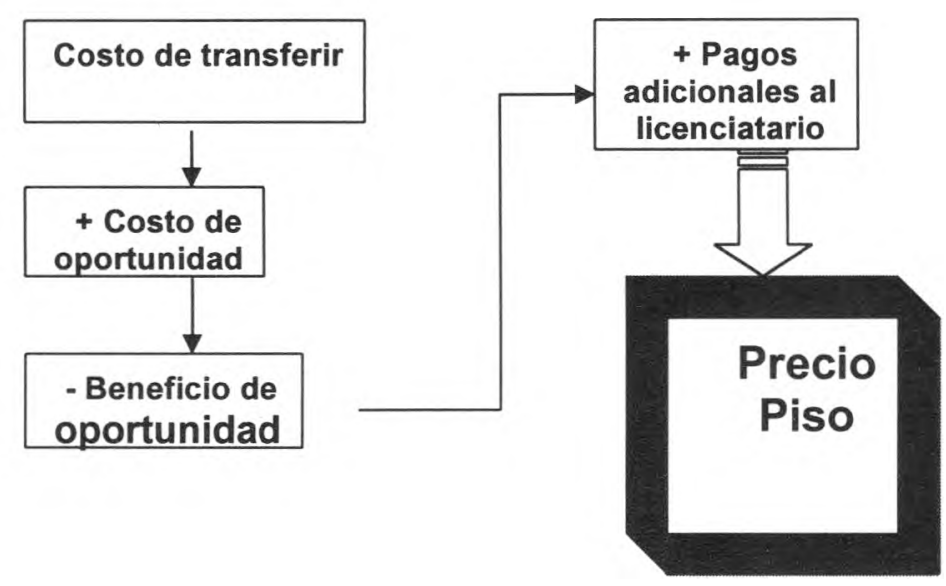

Figura 9. Criterio del costo minimo para definir el precio piso

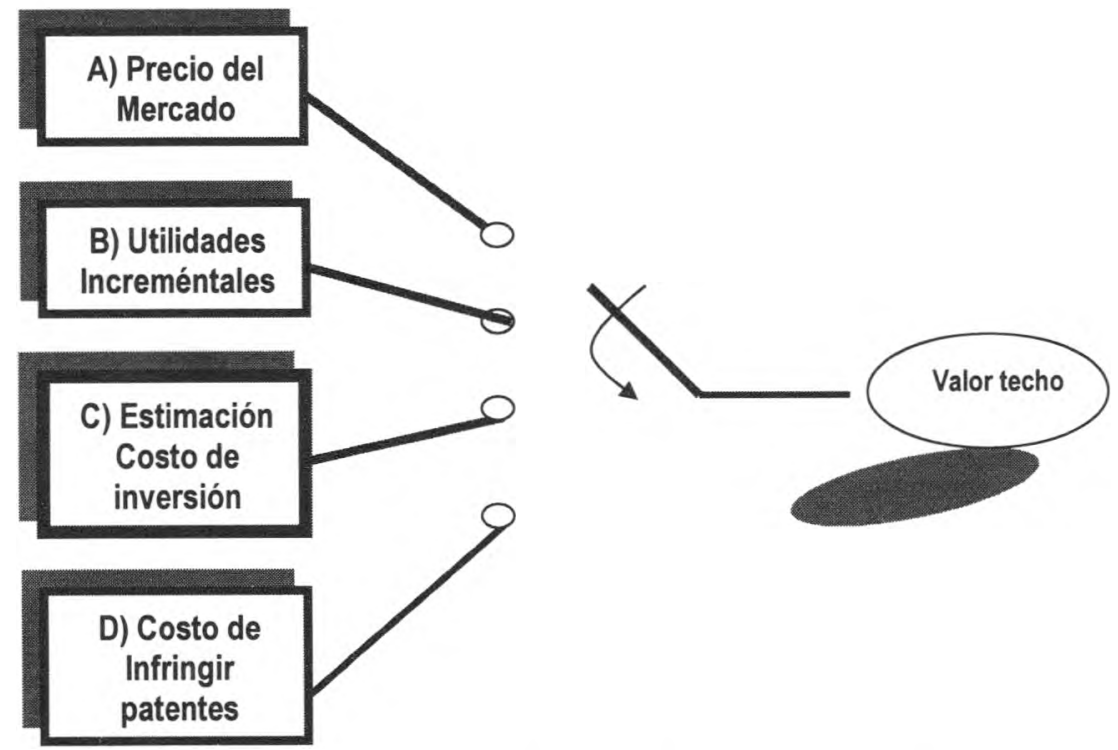

Figura 10. Abanico de alternativas para el avalúo techo

Un caso típico son los desarrollos logrados en instituciones públicas de investigación y desarrollo, financiados por fondos públicos. En estos casos la transferencia de tecnología puede verse como una oportunidad no esperada.

El costo de transferir se calcula como el total de los gastos de administración, salarios del personal, viajes, derechos de propiedad intelectual, etc. A esto se le agrega el costo de oportunidad como los materiales y el costo del personal para finiquito de prototipos y muestras, estudios de mercado, investigación financiera de la contraparte, pruebas de laboratorio, así como todo aquel costo adicional que pudiera presentarse.

A la cantidad anterior hay que deducirle los posibles beneficios de oportunidad. En algunas negociaciones se pueden obtener trueques, se puede disponer de maquinaria, equipos y personal especializado, así como 
de capacitación. Algunas veces existen otros beneficios políticos indirectos, los cuales hay que cuantificar. Finalmente, hay que agregar algún posible pago al licenciatario originado por negociaciones previas entre las partes.

\section{Paso 3. Avalúo techo}

La figura 10 muestra el abanico de alternativas para la definición del valor máximo a cobrar, es decir, del avalúo techo

\section{A). Avalúo techo por precio del mercado}

Este avalúo puede realizarse cuando la tecnología se encuentre en las zonas de máxima y mínima incertidumbre. Existen dos casos posibles:

Caso 1. Tecnologías en la zona 3 o de mínima incertidumbre. En este caso la tecnología es conocida, cuenta con un mercado común, existen datos de ventas y de su comportamiento financiero. Es posible determinar los flujos de efectivo, así como proyectar el estado de resultados y la razón de utilidades futuras para un período de entre tres y diez años, y de esta forma trasladarlos al presente. Este procedimiento proporcionará una medida del valor presente de la tecnología. Usando este procedimiento es posible la valuación de empresas completas cuando se consid era el precio corriente de los activos, los costos de capital bajo un cierto riesgo y el agregado del valor de las oportunidades de crecimiento (Ferris et al., 1995).

Caso 2. Tecnologías en la zona 1 o de máxima incertidumbre. Este es el caso del otro extremo, generalmente de tecnologías en desarrollo. Se presenta cuando la tecnología es muy novedosa y requerida, por lo que se consideran innovaciones de ruptura de enormes beneficios. Algunos ejemplos pueden ser la vacuna contra el Sida, la cura de la calvicie, super conductores a temperatura ambiente, etc. En este caso se hacen estudios y estimaciones del precio que la contraparte estará dispuesta a pagar.

El mercado pone el precio y para determinarlo, se usan procedimientos de inteligencia tecnológica basadas en las ofertas realizadas por diferentes postores. Este caso es poco común.

\section{B). Avalúo techo por utilidades incrementales}

Este es un caso particular de tecnologías que se encuentran en la zona 3 de mínima incertidumbre. Se presenta cuando hay nuevas tecnologías consistentes en procesos, productos o equipos intermedios que se van a adaptar a la producción que ya se tiene y de los cuales se espera una mejora en la productividad, así comoen las utilidades.

Otro caso se observa cuando los nuevos procesos mejorados permiten la fabricación de otros productos, haciendo uso de la capacidad instalada y de la gente, generando utilidades incrementales sin incurrir en grandes costos adicionales.

El valor de la tecnología se determina estableciendo una tendencia de crecimiento para los aspectos financieros, después hay que proyectar el estado de resultados y la razón de utilidades para un período de mediano plazo y trasladarlos al presente. Este procedimiento proporcionará una medida del valor presente de la tecnología. Dadas las condiciones con- 
troladas de la situación, el margen de error en la valuación es muy bajo.

\section{C). Avalúo techo por estimación costo/inversión}

Este es el caso más común que se presenta cuando las tecnologías están en la zona 2 de Incertidumbre moderada.

Dos situaciones son posibles:

Caso 1. Se presenta cuando los productos tecnológicos están en la fase de introducción y crecimiento. Hay que realizar la evaluación financiera costo/beneficio en términos de estimaciones y proyecciones de los valores esperados de las ventas, asícomo calificar la utilidad esperada en valor presente. Este avalúo depende mucho del pronóstico de ventas. Los indicadores financieros son estimados porque en esta etapa del ciclo de vida no se conoce el comportamiento ni la aceptación real del producto tecnológico en el mercado. Hay que tener mucho cuidado de que las proyecciones y tendencias sean realistas, ya que si las mismas se inflan puede llevar a resultados engañosos de la valoración.

Caso 2. Se presenta en el extremo derecho de la curva del ciclo de vida, es decir, en la fase de declinación. En este caso síes posible que la empresa desaparezca, por lo que se puede utilizar el método financiero de liquidación de activos para realizar el avalúo.

D). Avalúo techo por estimación del costo de infringir patentes

Aunque la infracción de patentes puede presentarse en cualquier momento del ciclo de vida de una tecnología, el caso más frecuente es cuando las tecnologías se encuentran, ya sea en la zona 1 de desarrollo, o bien, en la etapa de introducción y crecimiento.

Generalmente se trata de desarrollos de alta tecnología. En este caso quien desarrolla debe estar consciente de la posibilidad de invadir algunas reivindicaciones de patentes de su competencia. Esto se liga con trabajos de inteligencia tecnológica competitiva de alto nivel.

Este es el avalúo más problemático porque para su realización se deben conocer, entre otras cosas, los costos de desarrollo del competidor con la misma tecnología, el nivel de negocio que espera el competidor y finalmente estimar o imaginar la cantidad por la cual el competidor demandaría, y como resultado, tomar en cuenta los costos del litigio y otros costos asociados al mismo. A partir de estas estimaciones pueden usarse técnicas financieras de flujo de caja descontado para la estimación del precio (Goldscheider, 1996) y (McMahon, 2000). A continuación se presentan algunos ejemplos de casos de invasión de patentes y litigio, tomados de Parr (1992) Hatton (1987) y Yamasaki (1996), entre otros.

En 1976, Kodak con su cámara instantánea infringió derechos de doce patentes de Polaroid, las ventas de Kodak fueron de 10,000 MDD, mientras que las ventas de Polaroid fueron de 1000 MDD. Kodak pagó 454.20 MDD en compensación, más 455.2 MDD por intereses en 1990.

En Los Angeles California EUA, Stac Electronics obtuvo una sentencia favorable de $120 \mathrm{MDD}$ en contra de 
Microsoft por la invasión de la tecnología de compresión de Stac que duplica la cantidad de acceso remoto en Windows NTy Windows paragrupos detrabajo 3.11

Intel Corporation pagó en 1994 \$10 MDD a Cyrix Corporation por el fallo de una corte en Sherman Texas por los derechos de venta de clones (tarjetas armadas con microprocesadores 386 y 486 de Intel).

En 1997, Laboratorios Bio Genex fabricante de kits para el diagnóstico de cáncer fue acreditado con más de $\$ 400,000.00$ dólares por parte de BioTek Solutions quien infringió patentes tejidos antígenos de pretratamientos.

Cell Pro Incorporated, recibió el fallo de una corte de Delaware para el pago de \$7 MDD por la infracción de patentes para el tratamiento del cáncer, tecnología desarrollada por la Universidad John Hopkins, Licenciada a Beckton Dickinson Co.

\begin{abstract}
- En 1997, Viskase recibió más de $\$ 102$ MDD por la invasión de sus patentes de la tecnología de plásticos paraelenvasadodecarnes (polloy pavo). La compañía invasora fue American National Can Company
\end{abstract}

\section{Paso 4. Determinación del valor final}

El valor final se determina por medio del proceso de negociación, no hay reglas escritas para esto; sin embargo, una vez que se tienen los valores piso y techo que determinan el rango de negociación se puede proceder a un ajuste fino del avalúo, que permita presentar argumentos sólidos ante la mesa de negociación.

Para el ajuste final se pueden introducir algunos elementos adicionales auxiliares para la evaluación, como las variables tecnológica, impacto ambiental, beneficio social u otras que pudieran ser importantes, según el caso.

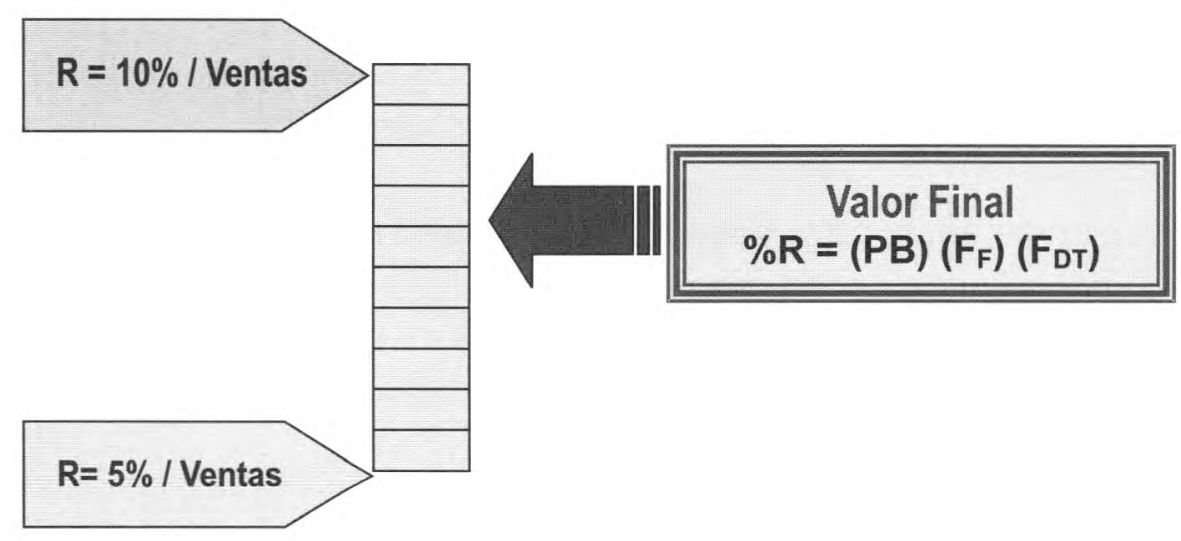

Figura 11. Ejemplo de determinación de valor final por medio de la modificación del parámetro base 
En la figura 11 puede verse el planteamiento de un ejemplo de aplicación del método general de valuación. Partimos de los siguientes supuestos: el valor inicial se considera igual al valor piso y es igual al $5 \%$ del valor de las ventas como regalías anuales. Esto fue definido por medio de las tablas de normas industriales.

Por otra parte, se considera que el valor techo será del $10 \%$ de regalías, ya que se estima que existe la tendencia de que las ventas del producto tecnológico y de las utilidades se incrementen al doble.

Finalmente, se utilizará el criterio de modificación del parámetro base o inicial de regalías para obtener el valor final. Los ajustes se realizarán por medio de la calificación de los aspectos financieros $\left(\mathrm{F}_{\mathrm{F}}\right)$ y por el desempeño tecnológico $\left(\mathrm{F}_{\mathrm{DT}}\right)$.

Las condiciones del problema son:

$\% \mathrm{R}_{\text {mínimo }}=5 \%$ de las ventas, $\% \mathrm{R}_{\text {máximo }}=$ $10 \%$ por lo que el rango de valores para los factores financiero y de desempeño tecnológico son: $\% \mathrm{R}_{\text {final }}=10 \%=(5 \%)\left(\mathrm{F}_{\mathrm{F}}\right)$ $\left(\mathrm{F}_{\mathrm{DT}}\right)=(5 \%)(2)=(5 \%)(1.4)(1.4)$.

Así el factor de ajuste por desempeño financiero $\left(\mathrm{F}_{\mathrm{F}}\right), 0<\mathrm{F}_{\mathrm{F}}<1.4$

Para el cálculo de $\mathrm{F}_{\mathrm{F}}$ se puede utilizar una variable intermedia " $\mathrm{x}$ ", se sugiere cualquiera de las siguientes posibilidades que hacen a la variable adimensional.
$\mathrm{X}=($ TIR del proyecto)/(TIR mínima aceptable), o bien

$\mathrm{X}=$ (Utilidad esperada por el proyecto)/ Utilidad mínima aceptable

El cálculo de la atractividad financiera se realiza mediante un escalamiento lineal para la variable, según se muestra en la tabla 2 .

Supóngase que para nuestro producto tecnológico mediante algunos pronósticos de ventas se identifica en que la utilidad anual esperada será del $80 \%$, mientras que la utilidad mínima aceptable en proyectos similares es de un 15\% arriba dela tasa anual de inversiones, al plazo fijo $\mathrm{U}_{\mathrm{MA}}=15 \%$ $+20 \%=35 \%$.

Así, $\mathrm{X}=80 \% / 35 \%=2.285$, por lo que la atractividad financiera de la tecnología es muy alta y el factor de ajuste según la tabla 2 es del 1.40

\section{Factor de ajuste por desempeño tecnológico $\left(\mathrm{F}_{\mathrm{DT}}\right) ; 0<\mathrm{F}_{\mathrm{DT}}<1.4$}

La tabla 3 muestra una matriz de ponderación auxiliar para calificar el desempeño tecnológico de la tecnología. La primera columna incluye los diversos factores que son importantes para la misma, según la opinión del analista evaluador.

Tabla 2. Escalamiento final para el cálculo de la atractividad financiera o factor de desempeño financiero

\begin{tabular}{ccc}
\hline Valor calculado de $\mathrm{X}$ & Atractividad de la tecnología & Calificación de $\mathrm{F}_{\mathrm{F}}$ \\
\hline$<1.0$ & No atractiva & 0.0 \\
De 1.0 a 1.3 & Baja & 0.35 \\
De 1.3 a 1.6 & Media & 0.70 \\
De 1.6 a 2.0 & Alta & 1.05 \\
$>2.0$ & Muy atractiva & 1.40 \\
\hline
\end{tabular}


En la segunda columna se asigna una calificación para cada factor tecnológico, con un valor entre uno y cinco, asignado según la importancia relativa entre factores. Por ejemplo, la exclusividad no es importante para el producto tecnológico, por lo que se le asigna un valor de 2 unidades, sin embargo la flexibilidad es muy importante ya que se le asigna un valor de cinco. Por medio de la suma de valores de la columna dos, obtenemos ellímite inferior o calificación mínima de desempeño $\left(C_{d}\right.$ mínimo).

La tercera columna incluye la calificación que dan a la tecnología algunos expertos, a quienes se les informa del proyecto y se les documenta con información sobre todas las características de la tecnología. La cuarta columna se forma con el producto de las columnas dos y tres. La suma de valores de esta columna nos da la calificación de desempeño para la tecnología $\left(C_{d}\right)$.

Tabla 3. Matriz de ponderación para la determinación del $\mathrm{F}_{\mathrm{DT}}$

\begin{tabular}{|c|c|c|c|}
\hline Factor & $\begin{array}{l}\text { Peso relativo entre } \\
\text { factores de } 1 \text { a } 5\end{array}$ & Calificación de 1 a 5 & Total $C_{d}$ de 1 a 25 \\
\hline 1. Calidad de la tecnología & 4 & 4 & 16 \\
\hline 2. Dimensiones tecnológicas & 4 & 4 & 16 \\
\hline 3. Adaptabilidad & 5 & 3 & 15 \\
\hline 4. Complejidad & 5 & 4 & 20 \\
\hline 5. Grado de innovación & 3 & 5 & 15 \\
\hline $\begin{array}{l}\text { 6. Integración del paquete } \\
\text { tecnologico }\end{array}$ & 3 & 3 & 9 \\
\hline 7. Exclusividad & 2 & 2 & 4 \\
\hline 8. Grado de desarrollo & 3 & 3 & 9 \\
\hline $\begin{array}{l}\text { 9. Sistema de Asimilación } \\
\text { Tecnológica }\end{array}$ & 4 & 4 & 16 \\
\hline 10. Ciclo de vida & 3 & 2 & 6 \\
\hline \multirow[t]{2}{*}{ 11. Flexibilidad } & 5 & 4 & 20 \\
\hline & $\sum=L_{i}=C_{d \text { minimo }}=41$ & & $\sum=146=C_{d}$ \\
\hline
\end{tabular}

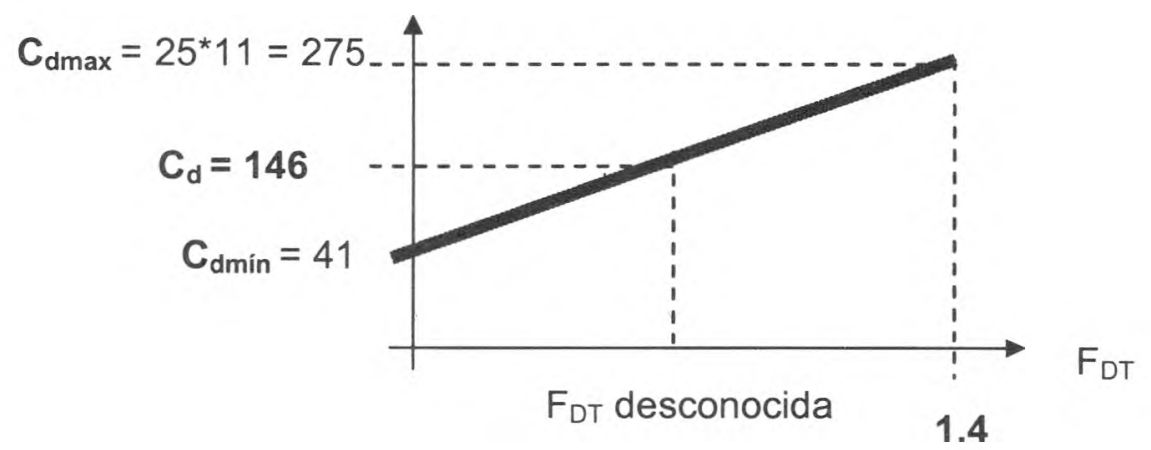

Figura 12. Transformación lineal para el factor de desempeño tecnológico 
El último paso es la traducción de la calificación de desempeño $C_{d}$ al factor de desempeño tecnológico $\mathrm{F}_{\mathrm{DT}}$. La figura 12 muestra la línea recta utilizada para la transformación lineal del $\mathrm{F}_{\mathrm{DT}}$.

Por medio de un cálculo sencillo se obtiene la $\mathrm{F}_{\mathrm{DT}}$ desconocida $=0.875$

El cálculo de regalías modificado es entonces:

$$
\begin{gathered}
\% R_{\mathrm{F}}=(\mathrm{PB})\left(\mathrm{F}_{\mathrm{F}}\right)\left(\mathrm{F}_{\mathrm{DT}}\right)=5 \% * 1.40 * 0.875 \\
=6.125 \%
\end{gathered}
$$

De esta forma, podremos entrar a la negociación de la tecnología con un valor de regalías preestablecido de $6.125 \%$ anual sobre las ventas.

\section{Conclusiones}

La determinación del precio de una tecnología debe ser un proceso creativo del cual no hay reglas escritas. Un buen avalúo contribuirá directamente con las negociaciones para lograr una transferencia de tecnología exitosa. La metodología presentada en este trabajo puede ser un auxiliar en el desarrollo de análisis para la determinación del precio.

Cada proyecto de avalúo requerirá de ajustes al método. Por ejemplo, si se trata de tecnologías en el área ambiental, el procedimiento mostrado podría extenderse para realizar la modificación del parámetro base de regalías, incluyendo esta variable. De esta forma, la expresión podría quedar $\% \mathrm{R}=(\mathrm{PB})\left(\mathrm{F}_{\mathrm{F}}\right)\left(\mathrm{F}_{\mathrm{DT}}\right)\left(\mathrm{F}_{\mathrm{A}}\right)$, donde $\mathrm{F}_{\mathrm{A}}$ es elfactor ambiental. Para identificar la variación de este factor en el tiempo, es posible usar la teoría de opciones, considerando que la tecnología puede tener un aumento o decremento en el precio, con respecto al tiempo en términos de otras variables relacionadas.

Pueden definirse funciones de cambio, precio, tipo exponencial, logarítmico, lineales, no lineales, cuadráticas, etcétera. Un caso sencillo sería tomar al factor ambiental como $F_{A}=\exp (\alpha t)$, donde el exponente puede ser positivo si el precio es incremental y negativo si el precio es decremental. Para obtener los valores, se requiere hacer estimaciones econométricas, pronósticos o hacer uso de gráficas de tendencias (Sunnenvag, 1998).

De acuerdo a los comentarios finales de la primera parte de este artículo se debe partir del mercado, en donde para definir con mayor precisión cuáles serán las herramientas más apropiadas para el avalúo, hay que iniciar por responder las siguientes preguntas ¿Quién es el cliente?, ¿Cuál es la relación que existe?, ¿Cuáles su percepción del mercado? y preferentemente realizar la determinación de los otros tres aspectos de evaluación, aspectos críticos, grado de integración del paquete y desempeño de la tecnología.

Es cierto que siempre existe la posibilidad de que se presenten eventos imponderables en los tiempos de desarrollo e introducción de las tecnologías, por lo que la estimación de precios puntuales siempre deberá revisarse y actualizarse según el avance en el desarrollo de los proyectos. La metodología presentada pretende ofrecer al analista algunas herramientas para eliminar la gran subjetividad existente en los procesos de avalúo y evaluación tecnológica. 


\section{Referencias}

Bidault F. (1989). Technology Pricing: from Principles to Strategy. St. Martin Press, N.Y. EUA

Blumeschein $K$ y Johannesson M. (1999). Use of Contingent Valuation to Place a Monetary Value on Pharmacy Services. Clinical Therapeutics, Vol. 21, Issue 8, August 1999, 140-147. Colmer G., Dunkley M., Gray K., Pugh P. y Williamson A. (1999). Estimating the Cost of New Technology Products. International Journal of Technology Management, Vol. 17, Nos. 7/8, 840-846. Drucker A.G., Gómez V., Anderson S. (2001). Environmental Economic Valuation Methodologies. Ecological Economics, Vol. 36, Issue 1, January 2001, 1-18.

Everett M.R. (1995). Diffusion of Innovations. The Free Press, USA.

Ferris S.P., Joshi Y.P., Makhija A.K. (1995). Valuing an east European Company. Long Range Planning, Vol. 28, No. 6, 48-60. Elsevier Science Ltd.

Goldscheider R. (1996). Royalties as Measure of Damages. Les Nouvelles, Vol. XXXI, No.3, 115-120.

Hatton R. (1987). Licensing Specialty Chemicals. Specialty Chemicals, Vol. 7 No. 6, 392-395.

Kotler P. (1985). Fundamentos de mercadotecnia. Prentice Hall Hispanoamericana, S.A.

Levitt T. (1965). Exploit the Product Life Cycle. Harvard Business Review, 93-105. Martin J.W. (2000). Cost Effective Use of Evaluation Models: An Empirical Cross-National Investigation. International Journal of Technology Management, Vol. 19, Nos. 3/4/5, 368-383. Elsevier.
McGavock D., Haas D.A., Patin M.P. (1992). Factors Affecting Licensing Rates. Les Nouvelles, No. 3 Vol. XXVII, $107-112$.

McMahon J. (2000). Royalty Apportionment and the Discounted Cash Flow Model. Licensing Economics Review, No. 2000-2, 9-12, April.

Newton D.P, Pearson A.W. (1994). Application of Option Pricing Theory to R\&D. ReD Management, Basil Blackwell Ltd. Vol. 24, No.1, 83-89.

Obbard E.G. (1992). Technology Valuations for Cross-licensing. Licensing Economics Review, Vol. 3, No. 1, 12-19, October.

Parr L.R., Ed. (1992). Pharmaceutical Companies \& Economic Royalty Rates. Licensing Economics Review, Vol. 2, No. 5 April, 12-16.

Premachandra Wttage, Smith A., Pitts C., McDonald A. y Key D. (2000). Integrating Environment Impact, Contingent Valuation and Cost-Benefit Analysis \& Empirical Evidence for an Alternative Perspective. Impact Assessment $\theta$ Project Appraisal, Vol. 18 No. 1. 5-14. Beech Tree Publishers.

Pride W.M. y Ferrel O.C. (1986). Marketing, decisiones y conceptos básicos. Editorial Interamericana, 183.

Rodríguez D. y Vega L.R. (1995). El avalúo en la transferencia de tecnología de la universidad a la industria. Anales del VI Seminario Latinoamericano ALTEC, 1995, Concepción Chile, Vol. I, 407-425.

Rodríguez D. y Solleiro J.L. (1991). Selección y avalúo de tecnologías, dos elementos básicos para la negociación. IV Seminario Latinoamericano de Gestión Tecnológica: competitividad, 
DOI: http://dx.doi.org/10.22201/fi.25940732e.2003.04n4.017

Evaluación, avalúo y ciclo de vida de la tecnología (Parte II)

reconversión industrial y tecnología, Caracas Venezuela, 191-200, Septiembre.

Schewe D.C. y Smith R.M. (1988). Mercadotecnia, conceptos y aplicaciones, Mc Graw Hill, México.

Shippley D. y Jobber D. (2001). Integrative Pricing Via the Pricing Wheel. Industrial Marketing Management, Vol. 30, Issue 3, April, 301-314.

Stephen P.F. y Yash P.J. (1995). Valuing an East European Company; Long Range Planning; Vol. 28, No. 6, 48-60, Elsevier Pergamon, GB.

Sullivan P.H. (1994). Royalty Rates Conform Industry Norm. Les Nouvelles, Vol. XXIX, No. 3, 140-146.

Stirling A. (1997). Limits to the Value of External Cost. Energy Policy, Vol.25, No. 5, 517-540, Elsevier Science Limited, GB.

Sunnevag K. (1998). An Option Pricing Approach to Exploration Licensing
Strategy. Resources Policy, Vol. 24, No. 1, 25-38, Great Britain.

UNIDO (1996). Manual on Technology Transfer Negotiation. United Nations Industrial Development Organisation, Vienna.

Washington Research Foundation. (1992). Valuing the Technology. Technology Fellowships Program Handbook.

Whittington D. (1998). Administering Contingent Valuation Surveys in Developing Countries. World Development, Vol. 26, Issue 1, 21-30.

Willis K.G., Garrod G.D. y Harvey D.R. (1998). A Review of Cost-benefit Analysis to the Evaluation of New Road Proposals in the U.K. Transportation Research Part D: Transport e Environment, Vol. 3, Issue 3, May 1998, 141-156.

Yamasaki M. (1996).. Determining Pharmaceutical Royalties. Les Nouvelles, Septiembre, 112-115.

\section{Semblanza del autor}

Luis Roberto Vega-González. Es ingeniero mecánico electricista por la Facultad de Ingeniería de la UNAM. Obtuvo la maestría en ingeniería de sistemas en el área de planeación por la DEPFI-UNAM y la maestría en administración de las organizaciones por la División de Estudios de Posgrado de la Facultad de Contaduría y Administración de la UNAM. Asimismo, se especializó en gestión de la innovación tecnológica por el Centro para la Innovación Tecnológica y la FCyA de la UNAM. Durante más de veinte años colaboró en diversas firmas nacionales e internacionales con proyectos en las áreas de instrumentación, control y potencia. Actualmente es coordinador de vinculación y gestión tecnológica del Centro de Ciencias Aplicadas y Desarrollo Tecnológico (CCADET) de la UNAM. 